\title{
The number of cube-full numbers in an interval
}

by

\author{
Hong-QuAN LiU (Harbin)
}

1. Introduction. A positive integer $n$ is called cube-full if $p \mid n$ implies that also $p^{3} \mid n$; here $p$ denotes a prime number. Let $Q_{3}(x)$ denote the number of cube-full numbers in the interval $[1, x]$, where $x$ is a sufficiently large number. On the one hand, an asymptotic formula for $Q_{3}(x)$ can be given with an error term $o\left(x^{1 / 8}\right)$, and this order of magnitude cannot be reduced to $O\left(x^{\mu}\right)$ with some $\mu<1 / 8$ without any hypothesis. On the other hand, an asymptotic formula does hold for $Q_{3}\left(x+x^{2 / 3+\mu}\right)-Q_{3}(x)$ with a $\mu<1 / 8$ by using results for exponential sums. In fact, Shiu [7] proved that

(*) $\quad Q_{3}\left(x+x^{2 / 3+\mu}\right)-Q_{3}(x) \rightarrow C x^{\mu}, \quad$ for any $\frac{140}{1123}<\mu<\frac{1}{3}$;

here $140 / 1123=0.1246607 \ldots(<1 / 8)$. The aim of this paper is to give an improvement on his result. We will prove

Theorem 1. (*) holds for

$$
\frac{1}{3}>\mu>\frac{11}{92}=0.1195652 \ldots
$$

Taking the idea from the author's paper [3] dealing with squarefull integers, we first give a reduction of our problem, which connects $(*)$ with some exponential sums, but this time (in contrast to [3]) triple exponential sums are inevitable, and, to obtain our result, it is crucial to apply the method developed in [2] and [4]. Actually, we also give an improvement of Krätzel's result about $\Delta(x ; 3,4,5)$, where $\Delta(x ; 3,4,5)$ is the error term in the asymptotic formula

$$
\begin{gathered}
\sum_{x \geq r^{3} s^{4} t^{5}} 1=A_{3}^{*} x^{1 / 3}+A_{4}^{*} x^{1 / 4}+A_{5}^{*} x^{1 / 5}+\Delta(x ; 3,4,5), \\
A_{i}^{*}=\prod_{\substack{3 \leq n \leq 5 \\
n \neq i}} \zeta\left(\frac{n}{i}\right), \quad i=3,4,5 .
\end{gathered}
$$


Our estimates then imply $\Delta(x ; 3,4,5) \ll x^{11 / 92+\varepsilon}$, where $\varepsilon$ is an arbitrarily small positive number, while [1] finds $\Delta(x ; 3,4,5) \ll x^{22 / 177+\varepsilon}$, with $22 / 177=0.1242937 \ldots$

2. Reduction. We prove

TheOREM 2. Let

$$
S_{a, b, c}(x)=\sum_{(m, n) \in D} \psi\left(\left(\frac{x}{m^{b} n^{c}}\right)^{1 / a}\right), \quad \psi(\xi)=\xi-[\xi]-\frac{1}{2},
$$

where $(a, b, c)$ is any permutation of $(3,4,5), D$ is the range $\left\{(m, n) \mid m^{a+b} n^{c}\right.$ $\leq x, m \geq n\}$ or $\left\{(m, n) \mid m^{a+b} n^{c} \leq x, m \geq x^{1 / 12}\right\}$ or $\{(m, n) \mid n \leq m \leq$ $\left.x^{1 / 12}\right\}$. Then

$$
S_{a, b, c}(x) \ll x^{11 / 92+\varepsilon}
$$

implies the assertion of Theorem 1.

Proof. Put $B=x^{\varepsilon}$, and suppose that $11 / 92+\varepsilon<\mu<1 / 3$. It is well known that

$$
Q_{3}(x)=\sum_{\substack{a^{3} b^{4} c^{5} \in I \\ b, c \leq B}}|\mu(b c)|+\sum_{\substack{a^{3} b^{4} c^{5} \in I \\ b>B \text { or } c>B}}|\mu(b c)|=\sum_{1}+\sum_{2}, \text { say, }
$$

where $I$ is the interval $\left(x, x+x^{2 / 3+\mu}\right]$. We have

$$
\begin{gathered}
\sum_{1}=\left(\left(x+x^{2 / 3+\mu}\right)^{1 / 3}-x^{1 / 3}\right) \sum_{b \leq B} \frac{|\mu(b)|}{b^{4 / 3}} \sum_{\substack{(c, b)=1 \\
c \leq B}} \frac{|\mu(c)|}{c^{5 / 3}}+O\left(B^{2}\right), \\
\sum_{\substack{(c, b)=1 \\
c \leq B}} \frac{|\mu(c)|}{c^{5 / 3}}=\sum_{\substack{c=1 \\
(c, b)=1}}^{\infty} \frac{|\mu(c)|}{c^{5 / 3}}+O\left(B^{-2 / 3}\right) \\
=\frac{\zeta(5 / 3)}{\zeta(10 / 3)} \prod_{p \mid b}\left(1+\frac{1}{p^{5 / 3}}\right)^{-1}+O\left(B^{-2 / 3}\right), \\
\sum_{b \leq B} \frac{|\mu(b)|}{b^{4 / 3}} \prod_{p \mid b}\left(1+\frac{1}{p^{5 / 3}}\right)^{-1}=\prod_{p}\left(1+\frac{1}{p^{4 / 3}}\left(1+\frac{1}{p^{5 / 3}}\right)^{-1}\right)+O\left(B^{-1 / 3}\right), \\
\left(x+x^{2 / 3+\mu}\right)^{1 / 3}-x^{1 / 3}=\frac{1}{3} x^{\mu}\left(1+O\left(x^{2 \mu-2 / 3}\right)\right),
\end{gathered}
$$

and thus

$$
\sum_{1}=C x^{\mu}(1+o(1)), \quad C=\frac{1}{3} \cdot \frac{\zeta(5 / 3)}{\zeta(10 / 3)} \prod_{p}\left(1+\frac{1}{p^{4 / 3}}\left(1+\frac{1}{p^{5 / 3}}\right)^{-1}\right) .
$$


It remains to estimate $\sum_{2}$. First we consider the portion of $\sum_{2}$ with $b>B$, which we denote by $\sum_{21}$. We have, with $x_{1}=x+x^{2 / 3+\mu}$ and $a(m)=\sum_{m=a^{3} c^{5}} 1$,

$$
\begin{aligned}
\sum_{21} \leq & \sum_{B<b \leq x_{1}^{1 / 12}} \sum_{\left(x b^{-4}\right)^{1 / 3}<a c^{5 / 3} \leq\left(x b_{1} b^{-4}\right)^{1 / 3}} 1 \\
& +\sum_{m \leq x_{1}^{2 / 3}} a(m) \sum_{\left(x m^{-1}\right)^{1 / 4}<b \leq\left(x_{1} m^{-1}\right)^{1 / 4}} 1 .
\end{aligned}
$$

At this stage, to treat the sums of $(* *)$ we need to cite the next two lemmata.

LEMmA 2.1. For $y \geq 1, \varrho>0,1 \leq \mu \leq y^{1 /(\varrho+1)} \leq v, \mu^{\varrho} v=y$,

$$
\begin{array}{r}
\sum_{y^{1 /(\varrho+1)<n \leq v}} \psi\left(\sqrt[\varrho]{\frac{y}{n}}\right)=\sum_{\mu<n \leq y^{1 /(\varrho+1)}} \psi\left(\frac{y}{n^{\varrho}}\right)-\varrho \psi_{2}(\mu) y \mu^{-1-\varrho} \\
+O\left(y \mu^{-2-\varrho}+1\right),
\end{array}
$$

where $\psi_{2}(z):=\frac{1}{2} \psi(z)^{2}-1 / 24$.

LEMma 2.2. With the same assumptions as in Lemma 2.1,

$$
\begin{array}{r}
\sum_{m^{\varrho} n \leq y} 1=\zeta(\varrho) y+\zeta\left(\frac{1}{\varrho}\right) y^{1 / \varrho}-\sum_{n \leq \mu} \psi\left(\frac{y}{n^{\varrho}}\right)-\sum_{n \leq v} \psi\left(\sqrt[\varrho]{\frac{y}{n}}\right)-\varrho \psi_{2}(\mu) y \mu^{-1-\varrho} \\
+O\left(y \mu^{-2-\varrho}\right)+1 / 4-\psi(\mu) \psi(v)+O\left(\mu v^{-1}\right) .
\end{array}
$$

Lemma 2.1 is Hilfssatz 4 of P. G. Schmidt [6], and Lemma 2.2 is (8) on p. 37 of [6]. Now by Lemma 2.2 we have, with $\mu=\left(x_{1} b^{-4}\right)^{1 / 8}$,

$$
\begin{aligned}
\sum_{a c^{5 / 3} \leq\left(x_{1} b^{-4}\right)^{1 / 3}} 1= & \zeta\left(\frac{5}{3}\right)\left(x_{1} b^{-4}\right)^{1 / 3}+\zeta\left(\frac{3}{5}\right)\left(x_{1} b^{-4}\right)^{1 / 5} \\
& -\sum_{n \leq \mu} \psi\left(\left(x_{1} b^{-4} n^{-5}\right)^{1 / 3}\right) \\
& -\sum_{n \leq \mu} \psi\left(\left(x_{1} b^{-4} n^{-3}\right)^{1 / 5}\right)+O(1)
\end{aligned}
$$

and a similar formula holds for $x_{1}$ being replaced by $x$. Thus the first sum of $(* *)$ is (note that we can assume, for example, $\mu<0.125$, in light of [7])

$$
\leq O\left(B^{-1 / 3} x^{\mu}\right)+S_{1}
$$

where $S_{1}$ is a linear combination of $S_{a, b, c}(\Omega), \Omega=x$ or $x_{1}$, and $(a, b, c)$ belongs to the set $\{(3,4,5),(3,5,4),(5,4,3),(5,3,4)\}$. To deal with the second sum in $(* *)$ we apply the technique in Schmidt [6]. First it is easy to observe 
that

$$
\sum_{m \leq x_{1}^{2 / 3}} a(m) \sum_{\left(x m^{-1}\right)^{1 / 4}<b \leq\left(x_{1} m^{-1}\right)^{1 / 4}} 1 \leq I_{1}+I_{2},
$$

where

$$
\begin{aligned}
I_{1} & =\sum_{a^{3} c^{5} \leq x_{1}^{2 / 3}, a \leq x_{1}^{1 / 12}} \sum_{\left(x a^{-3} c^{-5}\right)^{1 / 4}<b \leq\left(x_{1} a^{-3} c^{-5}\right)^{1 / 4}} 1, \\
I_{2} & =\sum_{a^{3} c^{5} \leq x_{1}^{2 / 3}, c \leq x_{1}^{1 / 12}} \sum_{\left(x a^{-3} c^{-5}\right)^{1 / 4}<b \leq\left(x_{1} a^{-3} c^{-5}\right)^{1 / 4}} 1 .
\end{aligned}
$$

It is easy to see that

$$
\begin{aligned}
I_{1} & =O\left(x^{\mu-\varepsilon}\right)+S\left(x_{1}\right)-S(x), \\
S\left(x_{1}\right) & =\sum_{a \leq x_{1}^{1 / 12}} \sum_{c \leq\left(x_{1}^{2 / 3} a^{-3}\right)^{1 / 5}} \psi\left(\left(\frac{x_{1}}{a^{3} c^{5}}\right)^{1 / 4}\right)
\end{aligned}
$$

and $S(x)$ is defined similarly. We have

$$
\begin{aligned}
\sum_{c \leq\left(x_{1}^{2 / 3} a^{-3}\right)^{1 / 5}} \psi\left(\left(\frac{x_{1}}{a^{3} c^{5}}\right)^{1 / 4}\right) & \\
& =\left(\sum_{c \leq\left(x_{1} a^{-3}\right)^{1 / 9}}+\sum_{\left(x_{1} a^{-3}\right)^{1 / 9}<c \leq\left(x_{1}^{2 / 3} a^{-3}\right)^{1 / 5}}\right) \psi(\cdot) \\
& =I_{3}+I_{4}, \quad \text { say. }
\end{aligned}
$$

From $I_{3}$ we can get sums of the type $S_{a, b, c}\left(x_{1}\right)$ easily, and for $I_{4}$ we choose $y=\left(x_{1} a^{-3}\right)^{1 / 5}, \varrho=4 / 5, \mu=x_{1}^{1 / 12}, v=\left(x_{1}^{2 / 3} a^{-3}\right)^{1 / 5}$ in Lemma 2.1, to obtain

$$
I_{4}=\sum_{x_{1}^{1 / 12}<n \leq\left(x_{1} a^{-3}\right)^{1 / 9}} \psi\left(\left(\frac{x_{1}}{a^{3} n^{4}}\right)^{1 / 5}\right)+O\left(y \mu^{-1-\varrho}+1\right) .
$$

Thus from $I_{4}$ we also get sums of the type $S_{a, b, c}(\Omega)$, with a permissible error. Similarly we can treat $I_{2}$. The other portion of $\sum_{2}$ with the condition $c>B$ can be treated along the same lines by using Lemmata 2.1 and 2.2. Hence our problem is reduced to treating a linear combination of sums of the type $S_{a, b, c}(\Omega)$. The proof of Theorem 2 can thus been finished.

3. Three general estimates for $S_{a, b, c}(x)$. Clearly we can assume that

$$
D=\left\{(m, n) \mid m^{a+b} n^{c} \leq x, m \geq n\right\} ;
$$

the other two cases can be treated similarly and more easily. We need 
Lemma 3.1. Let $H \geq 1, X \geq 1, Y \geq 1000$, let $\alpha, \beta$ and $\gamma$ be real numbers such that $\alpha \gamma(\gamma-1)(\beta-1) \neq 0$, and for $A>C(\alpha, \beta, \gamma)>0$, let $f(h, x, y)=A h^{\alpha} x^{\beta} y^{\gamma}$. Define

$$
S(H, X, Y)=\sum_{(h, x, y) \in D} C_{1}(h, x) C_{2}(y) e(f(h, x, y)),
$$

where $D$ is a region contained in the rectangle

$\{(h, x, y) \mid h \sim H, x \sim X, y \sim Y\} \quad(h \sim H$ means that $H \leq h<2 H$, etc. $)$

such that for any fixed pair $\left(h_{0}, x_{0}\right)$, the intersection $D \cap\left\{\left(h_{0}, x_{0}, y\right) \mid y \sim\right.$ $Y\}$ has at most $O(1)$ segments. Also, suppose $\left|C_{1}(h, x)\right| \leq 1,\left|C_{2}(y)\right| \leq 1$, $F=A H^{\alpha} X^{\beta} Y^{\gamma} \gg Y$. Then

$$
\begin{aligned}
L^{-3} S(H, X, Y) \ll & \sqrt[22]{(H X)^{19} Y^{13} F^{3}}+H X Y^{5 / 8}\left(1+Y^{7} F^{-4}\right)^{1 / 16} \\
& +\sqrt[32]{(H X)^{29} Y^{28} F^{-2} M^{5}}+\sqrt[4]{(H X)^{3} Y^{4} M}
\end{aligned}
$$

where $L=\ln (A H X Y+2), M=\max \left(1, F Y^{-2}\right)$.

LEMmA 3.2. Let $f(x, y)$ be an algebraic function in the rectangle $D_{0}=$ $\{(x, y) \mid x \sim X, y \sim Y\}, f(x, y)=A x^{\alpha} y^{\beta}$ for $(x, y) \in D_{0}, D$ be a subdomain of $D_{0}$ bounded by $O(1)$ algebraic curves. Suppose that $X \gg Y, N=X Y$, $A>0, F=A X^{\alpha} Y^{\beta}$, and $\alpha \beta(\alpha+\beta-1)(\alpha+\beta-2) \neq 0$. Then

$$
\begin{aligned}
S & :=(N F)^{-\varepsilon} \sum_{(x, y) \in D} e(f(x, y)) \\
& \ll \sqrt[6]{F^{2} N^{3}}+N^{5 / 6}+\sqrt[8]{N^{8} F^{-1} X^{-1}}+N F^{-1 / 4}+N Y^{-1 / 2} .
\end{aligned}
$$

Lemma 3.1 is Theorem 3 of [4], and Lemma 3.2 is Lemma 9 of [2]. We also need

LEMMA 3.3. Let $f(x)$ and $g(x)$ be algebraic functions in the interval $[a, b]$, and

$$
\begin{gathered}
\left|f^{\prime \prime}(x)\right| \cong R^{-1}, \quad\left|f^{\prime \prime \prime}(x)\right| \ll(R U)^{-1} \\
|g(x)| \leq H, \quad\left|g^{\prime}(x)\right| \ll H U_{1}^{-1}, \quad U, U_{1} \geq 1 .
\end{gathered}
$$

Then

$$
\begin{aligned}
\sum_{a \leq n \leq b} g(n) e(f(n))= & \sum_{\alpha \leq u \leq \beta} b_{u} \frac{g(n(u))}{\sqrt{f^{\prime \prime}(n(u))}} e(f(n(u))-u n(u)+1 / 8) \\
& +O\left(H \ln (\beta-\alpha+2)+H(b-a+R)\left(U^{-1}+U_{1}^{-1}\right)\right) \\
& +O\left(H \min \left(R^{1 / 2}, \max (1 /\langle\alpha\rangle, 1 /\langle\beta\rangle)\right)\right)
\end{aligned}
$$

where $[\alpha, \beta]$ is the image of $[a, b]$ under the mapping $y=f^{\prime}(x), n(u)$ is determined by the equation $f^{\prime}(n(u))=u, b_{u}=1 / 2$ or 1 according as $u$ is 
one of $\alpha, \beta$ or not, $\langle x\rangle$ is defined by

$$
\langle x\rangle= \begin{cases}\|x\| & \text { if } x \text { is not an integer, }\|x\|=\min _{n}|x-n|, n \in \mathbb{Z}, \\ \beta-\alpha & \text { if } x \text { is an integer, }\end{cases}
$$

and $\sqrt{f^{\prime \prime}}>0$ if $f^{\prime \prime}>0, \sqrt{f^{\prime \prime}}=i \sqrt{\left|f^{\prime \prime}\right|}$ if $f^{\prime \prime}<0$.

Proof. This is Theorem 2.2 of [5].

Now we proceed to deal with $S_{a, b, c}(x)$. It suffices to estimate $S(M, N)$, where

$$
\begin{gathered}
S(M, N)=\sum_{(m, n) \in D} \psi\left(\left(x m^{-b} n^{-c}\right)^{1 / a}\right), \\
D=D(M, N)=\left\{(m, n) \mid m \sim M, n \sim N, m^{a+b} n^{c} \leq x, m \geq n\right\},
\end{gathered}
$$

and $M, N$ are any positive integers such that

$$
M N>x^{11 / 92}, \quad 2 M \geq N, \quad M^{a+b} N^{c} \leq x .
$$

Then, using the familiar reduction (cf. [2]), for a parameter $K \in[100, M N]$, we get, with $\eta=\varepsilon^{2}$, and some $H \leq K^{2}$,

$$
x^{-\eta} S(M, N) \ll M N K^{-1}+\min (1, K / H) \Phi(H, M, N),
$$

where

$$
\begin{gathered}
\Phi(H, M, N)=H^{-1} \sum_{h \sim H}\left|\sum_{(m, n) \in D} e(f(h, m, n))\right|, \\
f(h, m, n)=h\left(\frac{x}{m^{b} n^{c}}\right)^{1 / a} .
\end{gathered}
$$

At this stage, we can assume that $x$ is irrational. We apply Lemma 3.3 to the summation over $m$, and get, with $M_{1}=\max (M, n), M_{2}=$ $\min \left(\left(x n^{-c}\right)^{1 /(a+b)}, 2 M\right)$,

$$
\begin{aligned}
\sum_{M_{1} \leq m \leq M_{2}} e(f(h, m, n)) & \\
= & \sum_{U_{1}<u<U_{2}} C_{1}\left(x^{-1} h^{-a} n^{c} u^{2 a+b}\right)^{-1 /(2(a+b))} e\left(C_{2}\left(x h^{a} u^{b} n^{-c}\right)^{1 /(a+b)}\right) \\
& +O\left(\frac{M}{H F}+\ln x\right)+O\left(\min \left(\left(\frac{M^{2}}{H F}\right)^{1 / 2}, \frac{1}{U_{2}-h b / a}\right)\right. \\
& \left.+\sum_{1 \leq i \leq 2} \min \left(\left(\frac{M^{2}}{H F}\right)^{1 / 2}, \frac{1}{\left\|g\left(n, X_{i}\right)\right\|}\right)\right)+R(h, n),
\end{aligned}
$$

where $C_{1}, C_{2}, C_{3}, \ldots$ denote certain constants,

$$
U_{1}=\frac{h b}{a}\left(x n^{-c} M_{2}^{-a-b}\right)^{1 / a}, \quad U_{2}=\frac{h b}{a}\left(x n^{-c} M_{1}^{-a-b}\right)^{1 / a},
$$




$$
\begin{gathered}
g(n, X)=\frac{h b}{a}\left(x n^{-c} X^{-a-b}\right)^{1 / a}, \\
X_{1}=\max (n, M), \quad X_{2}=2 M, \quad F=\left(x M^{-b} N^{-c}\right)^{1 / a}, \\
R(h, n)=\left\{\begin{array}{l}
\frac{1}{2} C_{1}\left(x^{-1} h^{-a} n^{c} u^{2 a+b}\right)^{-1 /(2(a+b))} e\left(C_{2}\left(x h^{a} u^{b} n^{-c}\right)^{1 /(a+b)}\right) \\
0 \quad \text { otherwise. }
\end{array}\right.
\end{gathered}
$$

It is easy to see that

$$
\begin{gathered}
\sum_{n \sim N} \min \left(\left(\frac{M^{2}}{H F}\right)^{1 / 2}, \frac{1}{U_{2}-h b / a}\right) \ll x^{1 / 12} \ln x, \\
\sum_{n \in I} R(n, h) \ll\left(\frac{M^{2}}{H F}\right)^{1 / 2}\left(N^{4 / 6}\left(F H N^{-1}\right)^{1 / 6}+\frac{N}{F H}\right) \\
\ll x^{1 / 12} \quad(\text { note that } F \gg M, \ldots),
\end{gathered}
$$

where $I$ is some subinterval of $[N, 2 N]$. We consider

$$
\begin{aligned}
\Omega & =\sum_{n \sim N} \min \left(\left(\frac{M^{2}}{H F}\right)^{1 / 2}, \frac{1}{\left\|g\left(n, X_{1}\right)\right\|}\right) \\
& =\left(\sum_{n \leq M, n \sim N}+\sum_{n>M, n \sim N}\right) \min (A, B)=\Omega_{1}+\Omega_{2},
\end{aligned}
$$

where

$$
\begin{aligned}
\Omega_{1} & =\sum_{n \leq M, n \sim N} \min \left(\left(\frac{M^{2}}{H F}\right)^{1 / 2}, \frac{1}{\left\|g_{1}(n)\right\|}\right), \\
g_{1}(n) & =\frac{h b}{a}\left(x n^{-c} M^{-a-b}\right)^{1 / a}, \\
\Omega_{2} & =\sum_{n>M, n \sim N} \min \left(\left(\frac{M^{2}}{H F}\right)^{1 / 2}, \frac{1}{\left\|g_{2}(n)\right\|}\right), \\
g_{2}(n) & =\frac{h b}{a}\left(x n^{-c-a-b}\right)^{1 / a}=\frac{h b}{a}\left(x n^{-12}\right)^{1 / a} .
\end{aligned}
$$

As $g_{1}(y)$ is monotonic, and $g_{1}^{\prime}(y) \cong H\left(x N^{-c-a} M^{-b-a}\right)^{1 / a}$ for $y \sim N$, by Hilfssatz 4 of Krätzel [1] we have

$$
\begin{aligned}
\Omega_{1} \ll & \left(1+H\left(x N^{-c} M^{-c-a}\right)^{1 / a}\right) \\
& \times\left(\left(M^{2} H^{-1} F^{-1}\right)^{1 / 2}+H^{-1}\left(x^{-1} N^{c+a} M^{b+a}\right)^{1 / a} \ln x\right) \\
\ll & (H F)^{1 / 2}+x^{1 / 12} \ln x
\end{aligned}
$$

and similarly, $\Omega_{2} \ll(H F)^{1 / 2}+x^{1 / 12} \ln x$ (note that $\Omega_{2} \neq 0$ implies $N \gg M$ ), and 


$$
\sum_{n \sim N} \min \left(\left(\frac{M^{2}}{H F}\right)^{1 / 2}, \frac{1}{\left\|g\left(n, X_{2}\right)\right\|}\right) \ll(H F)^{1 / 2}+x^{1 / 12} \ln x .
$$

From the above observations, we achieve, after a double Abelian summation, the estimate

$$
\text { (1) } \begin{aligned}
\Phi(H, M, N) \ll & H^{-1}\left(\frac{M^{2}}{H F}\right)^{1 / 2} \sum_{h \sim H}\left|\sum_{(u, n) \in D^{\prime}} e\left(C_{2}\left(x h^{a} u^{b} n^{-c}\right)^{1 /(a+b)}\right)\right| \\
& +(H F)^{1 / 2}+x^{1 / 12} \ln x,
\end{aligned}
$$

where $D^{\prime}$ is a range contained in $\left\{(u, n) \mid n \sim N, C_{3} \leq H F /(u M) \leq C_{4}\right\}$, bounded by $O(1)$ algebraic curves. We will apply the next lemma to choose parameters optimally.

Lemma 3.4. Let $M>0, N>0, u_{m}>0, v_{n}>0, A_{m}>0, B_{n}>0$ $(1 \leq m \leq M, 1 \leq n \leq N)$, and let $Q_{1}$ and $Q_{2}$ be given nonnegative numbers, $Q_{1} \leq Q_{2}$. Then there exists an $Q \in\left[Q_{1}, Q_{2}\right]$ with

$$
\begin{aligned}
\sum_{1 \leq m \leq M} A_{m} Q^{u_{m}}+\sum_{1 \leq n \leq N} B_{n} Q^{-v_{n}} \ll & \sum_{1 \leq m \leq M} \sum_{1 \leq n \leq N}\left(A_{m}^{v_{n}} B_{n}^{u_{m}}\right)^{1 /\left(u_{m}+v_{n}\right)} \\
& +\sum_{1 \leq m \leq M} A_{m} Q_{1}^{u_{m}}+\sum_{1 \leq n \leq N} B_{n} Q_{2}^{-v_{n}} .
\end{aligned}
$$

This is Lemma 2 of [2]. Now if we apply Lemma 3.2 to the inner double sum in (1), put our estimate into (0), and choose $K$ optimally via Lemma 3.4, we get

\section{LEMMA 1.}

$$
x^{-\varepsilon} S(M, N) \ll \sqrt[8 a]{x^{2} M^{5 a-2 b} N^{5 a-2 c}}+\sqrt[11 a]{x^{3} M^{3 a-3 b} N^{10 a-3 c}}+x^{11 / 92} .
$$

If we choose $(h, x, y)=(h, u, n)$ in Lemma 3.1 , then we get an estimate for the triple exponential sum in (1). Putting it into (0), and choosing $K$ optimally via Lemma 3.4, we immediately get

LEMMA 2.

$$
\begin{aligned}
x^{-\varepsilon} S(M, N) \ll & \sqrt[30]{F^{11} M^{11} N^{21}}+\sqrt[24]{F^{8} M^{8} N^{18}}+\sqrt[20]{F^{4} M^{4} N^{21}} \\
& +\sqrt[40]{F^{11} M^{11} N^{36}}+\sqrt[45]{F^{16} M^{16} N^{31}} \\
& +\sqrt[5]{F^{2} M^{2} N^{3}}+\sqrt[4]{F M N^{4}}+x^{11 / 92}
\end{aligned}
$$

To obtain our last general estimate, we apply Lemma 3.3 to the summation over $n$ in (1), to get

$$
\begin{aligned}
\Phi(H, M, N) \ll & \frac{M N}{H^{2} F} \sum_{h \sim H}\left|\sum_{(u, v) \in D^{\prime \prime}} P(u) Q(v) e(G(u, v, h))\right| \\
& +(H F)^{1 / 2}+x^{1 / 12} \ln x,
\end{aligned}
$$


where $|P(u)| \leq 1,|Q(v)| \leq 1$, and $D^{\prime \prime}$ is a suitable domain contained in the rectangle $\left\{(u, v) \mid H F /(M u) \in\left[C_{3}, C_{4}\right], H F /(N v) \in\left[C_{5}, C_{6}\right]\right\}$. We then apply Lemma 3.1 to the sum of $(2)$ by choosing $(h, x, y)=(h, u, v)$, and taking $K$ optimally via Lemma 3.4 , we get

LEMMA 3.

$$
\begin{aligned}
x^{-\varepsilon} S(M, N) \ll & \sqrt[32]{F^{13} M^{13} N^{19}}+\sqrt[13]{F^{5} M^{5} N^{8}}+\sqrt[29]{F^{13} M^{13} N^{12}} \\
& +\sqrt[52]{F^{23} M^{23} N^{24}}+\sqrt[47]{F^{18} M^{18} N^{29}} \\
& +\sqrt[6]{F^{3} M^{3} N^{2}}+\sqrt[5]{F^{2} M^{2} N^{3}}+x^{11 / 92}
\end{aligned}
$$

4. Proof of Theorem 1. As $M \gg N$, we easily observe that

$$
F \ll\left(x M^{-4} N^{-5}\right)^{1 / 3},
$$

thus by Lemmata 1 and 3 we obtain

$$
\text { (3) } \begin{aligned}
x^{-\varepsilon} S(M, N) \ll & \ll \sqrt[24]{x^{2} M^{7} N^{5}}+\sqrt[33]{x^{3} N^{12}}+x^{11 / 92} \\
& +\sqrt[96]{x^{13} M^{-13} N^{-8}}+\sqrt[39]{x^{5} M^{-5} N^{-1}}+\sqrt[87]{x^{13} M^{-23} N^{-43}}+\sqrt[141]{x^{18} M^{-18} N^{-3}} \\
& +\sqrt[18]{x^{3} M^{-3} N^{-9}}+\sqrt[15]{x^{2} M^{-2} N^{-1}}+x^{11 / 92}
\end{aligned}
$$

and thus

$$
\begin{aligned}
x^{-\varepsilon} S(M, N) \ll & \sum_{1 \leq i \leq 11} R_{i}+\sqrt[87]{x^{13} M^{-13} N^{-29}}+\sqrt[156]{x^{23} M^{-23} N^{-43}} \\
& +\sqrt[6]{x M^{-1} N^{-3}}+x^{11 / 92}
\end{aligned}
$$

where

$$
\begin{aligned}
R_{1} & =\min \left(\sqrt[24]{x^{2} M^{7} N^{5}}, \sqrt[96]{x^{13} M^{-13} N^{-8}}\right) \\
& \ll\left(\sqrt[24]{x^{2}\left(M^{13} N^{8}\right)^{4 / 7}}\right)^{7 / 23}\left(\sqrt[96]{x^{13} M^{-13} N^{-8}}\right)^{16 / 23}=x^{11 / 92}, \\
R_{2} & =\min \left(\sqrt[24]{x^{2} M^{7} N^{5}}, \sqrt[39]{x^{5} M^{-5} N^{-1}}\right) \\
& \ll\left(\sqrt[12]{x M^{5} N}\right)^{4 / 17}\left(\sqrt[39]{x^{5} M^{-5} N^{-1}}\right)^{13 / 17}=x^{2 / 17}<x^{0.119}, \\
R_{3} & =\min \left(\sqrt[24]{x^{2} M^{7} N^{5}}, \sqrt[141]{x^{18} M^{-18} N^{-3}}\right) \\
& \ll\left(\sqrt[12]{x\left(M^{6} N\right)^{6 / 7}}\right)^{14 / 61}\left(\sqrt[47]{x^{6} M^{-6} N^{-1}}\right)^{47 / 61}=x^{43 / 366}<x^{0.118}, \\
R_{4} & =\min \left(\sqrt[24]{x^{2} M^{7} N^{5}}, \sqrt[15]{x^{2} M^{-2} N^{-1}}\right) \\
& \ll\left(\sqrt[12]{x\left(M^{2} N\right)^{2}}\right)^{2 / 7}\left(\sqrt[15]{x^{2} M^{-2} N^{-1}}\right)^{5 / 7}=x^{5 / 42}<x^{0.119},
\end{aligned}
$$




$$
\begin{aligned}
& R_{5}=\min \left(\sqrt[33]{x^{3} N^{12}}, \sqrt[96]{x^{13} M^{-13} N^{-8}}\right) \\
& \ll\left(\sqrt[11]{x N^{4}}\right)^{77 / 205}\left(\sqrt[96]{x^{13} N^{-21}}\right)^{128 / 205}=x^{73 / 615}<x^{0.119}, \\
& R_{6}=\min \left(\sqrt[11]{x N^{4}}, \sqrt[39]{x^{5} M^{-5} N^{-1}}\right) \\
& \ll\left(\sqrt[11]{x N^{4}}\right)^{11 / 37}\left(\sqrt[39]{x^{5} N^{-6}}\right)^{26 / 37}=x^{13 / 111}<x^{0.118}, \\
& R_{7}=\min \left(\sqrt[11]{x N^{4}}, \sqrt[87]{x^{13} M^{-13} N^{-29}}\right) \\
& \ll\left(\sqrt[11]{x N^{4}}\right)^{77 / 135}\left(\sqrt[87]{x^{13} N^{-42}}\right)^{58 / 135}=x^{47 / 405}<x^{0.117}, \\
& R_{8}=\min \left(\sqrt[11]{x N^{4}}, \sqrt[156]{x^{23} M^{-23} N^{-43}}\right) \\
& \ll\left(\sqrt[11]{x N^{4}}\right)^{363 / 675}\left(\sqrt[156]{x^{23} N^{-66}}\right)^{46 / 675}=x^{56 / 675}<x^{0.1}, \\
& R_{9}=\min \left(\sqrt[11]{x N^{4}}, \sqrt[47]{x^{6} M^{-6} N^{-1}}\right) \\
& \ll\left(\sqrt[11]{x N^{4}}\right)^{77 / 265}\left(\sqrt[47]{x^{6} N^{-7}}\right)^{188 / 265}=x^{31 / 265}<x^{0.117}, \\
& R_{10}=\min \left(\sqrt[11]{x N^{4}}, \sqrt[6]{x M^{-1} N^{-3}}\right) \\
& \ll\left(\sqrt[11]{x N^{4}}\right)^{11 / 17}\left(\sqrt[6]{x N^{-4}}\right)^{6 / 17}=x^{2 / 17}, \\
& R_{11}=\min \left(\sqrt[11]{x N^{4}}, \sqrt[15]{x^{2} M^{-2} N^{-1}}\right) \\
& \ll\left(\sqrt[11]{x N^{4}}\right)^{11 / 31}\left(\sqrt[15]{x^{2} N^{-3}}\right)^{20 / 31}=x^{11 / 93} .
\end{aligned}
$$

Hence we have, with $\theta=11 / 92$,

$$
\begin{aligned}
x^{-\varepsilon} S(M, N) \ll & \sqrt[87]{x^{13} M^{-13} N^{-29}}+\sqrt[156]{x^{23} M^{-23} N^{-43}} \\
& +\sqrt[6]{x M^{-1} N^{-3}}+x^{\theta}
\end{aligned}
$$

which, in conjunction with (3), gives

(6) $x^{-\varepsilon} S(M, N) \ll \sqrt[24]{x^{2} M^{7} N^{5}}+R_{7}+R_{8}+R_{10}+x^{\theta} \ll \sqrt[24]{x^{2} M^{7} N^{5}}+x^{\theta}$.

By Lemma 2 we have

(7) $x^{-\varepsilon} S(M, N) \ll \sqrt[90]{x^{11} M^{-11} N^{8}}+\sqrt[15]{x^{2} M^{-2} N^{-1}}+\sqrt[60]{x^{4} M^{-4} N^{43}}$

$$
+\sqrt[120]{x^{11} M^{-11} N^{53}}+\sqrt[12]{x M^{-1} N^{7}}+x^{\theta} .
$$

Note that actually $\sqrt[87]{x^{13} M^{-13} N^{-29}} \ll \sqrt[6]{x M^{-1} N^{-3}}$, thus from (5) we get

$$
x^{-\varepsilon} S(M, N) \ll \sqrt[156]{x^{23} M^{-23} N^{-43}}+\sqrt[6]{x M^{-1} N^{-3}}+x^{\theta} .
$$


From (7) and (8) we get

$$
x^{-\varepsilon} S(M, N) \ll \sqrt[90]{x^{11} M^{-11} N^{8}}+\sqrt[15]{x^{2} M^{-2} N^{-1}}+\sum_{1 \leq i \leq 6} S_{i}+x^{\theta},
$$

where

$$
\begin{aligned}
S_{1} & =\min \left(\sqrt[60]{x^{4} N^{39}}, \sqrt[156]{x^{23} N^{-66}}\right) \\
& \leq\left(\sqrt[60]{x^{4} N^{39}}\right)^{110 / 279}\left(\sqrt[156]{x^{23} N^{-66}}\right)^{169 / 279}=x^{43 / 372}<x^{0.116}, \\
S_{2} & =\min \left(\sqrt[60]{x^{4} N^{39}}, \sqrt[6]{x N^{-4}}\right) \\
& \leq\left(\sqrt[60]{x^{4} N^{39}}\right)^{40 / 79}\left(\sqrt[6]{x N^{-4}}\right)^{39 / 79}=x^{55 / 474}<x^{0.117} \\
S_{3} & =\min \left(\sqrt[120]{x^{11} N^{42}}, \sqrt[156]{x^{23} N^{-66}}\right) \\
& \leq\left(\sqrt[120]{x^{11} N^{42}}\right)^{110 / 201}\left(\sqrt[156]{x^{23} N^{-66}}\right)^{91 / 201}=x^{47 / 402}<x^{0.117}, \\
S_{4} & =\min \left(\sqrt[120]{x^{11} N^{42}}, \sqrt[6]{x N^{-4}}\right) \\
& \leq\left(\sqrt[120]{x^{11} N^{42}}\right)^{40 / 61}\left(\sqrt[6]{x N^{-4}}\right)^{21 / 61}=x^{43 / 366}<x^{0.118}, \\
S_{5} & =\min \left(\sqrt[12]{x N^{6}}, \sqrt[156]{x^{23} N^{-66}}\right) \\
& \leq\left(\sqrt[12]{x N^{6}}\right)^{11 / 24}\left(\sqrt[156]{x^{23} N^{-66}}\right)^{13 / 24}=x^{17 / 144}<x^{0.119}, \\
S_{6} & =\min \left(\sqrt[12]{x N^{6}}, \sqrt[6]{x N^{-4}}\right) \leq\left(\sqrt[12]{x N^{6}}\right)^{4 / 7}\left(\sqrt[6]{x N^{-4}}\right)^{3 / 7}=x^{5 / 42}<x^{\theta} .
\end{aligned}
$$

Thus we have

$$
x^{-\varepsilon} S(M, N) \ll \sqrt[90]{x^{11} M^{-11} N^{8}}+\sqrt[15]{x^{2} M^{-2} N^{-1}}+x^{\theta} .
$$

From (6), (8) and (9) we finally achieve

$$
x^{-\varepsilon} S(M, N) \ll \sum_{1 \leq i \leq 4} K_{i}+x^{\theta},
$$

where

$$
\begin{aligned}
K_{1} & =\min \left(\sqrt[24]{x^{2} M^{7} N^{5}}, \sqrt[156]{x^{23} M^{-23} N^{-43}}, \sqrt[90]{x^{11} M^{-11} N^{8}}\right) \\
& =\min \left(A_{1}, B_{1}, C_{1}\right) \leq A_{1}^{219 / 692} B_{1}^{232.5 / 692} C_{1}^{240.5 / 692}=x^{657 / 5536}, \\
K_{2} & =\min \left(\sqrt[24]{x^{2} M^{7} N^{5}}, \sqrt[156]{x^{23} M^{-23} N^{-43}}, \sqrt[15]{x^{2} M^{-2} N^{-1}}\right) \\
& =\min \left(A_{2}, B_{2}, C_{2}\right) \leq A_{2}^{168 / 530} B_{2}^{52 / 530} C_{2}^{310 / 530}=x^{189 / 1590},
\end{aligned}
$$




$$
\begin{aligned}
K_{3} & =\min \left(\sqrt[24]{x^{2} M^{7} N^{5}}, \sqrt[6]{x M^{-1} N^{-3}}, \sqrt[15]{x^{2} M^{-2} N^{-1}}\right) \\
& =\min \left(A_{3}, B_{3}, C_{3}\right) \leq A_{3}^{20 / 63} B_{3}^{3 / 63} C_{3}^{40 / 63}=x^{5 / 42} \\
K_{4} & =\min \left(\sqrt[24]{x^{2} M^{7} N^{5}}, \sqrt[6]{x M^{-1} N^{-3}}, \sqrt[90]{x^{11} M^{-11} N^{8}}\right) \\
& =\min \left(A_{4}, B_{4}, C_{4}\right) \leq A_{4}^{164 / 515} B_{4}^{111 / 515} C_{4}^{240 / 515}=x^{123 / 1030} .
\end{aligned}
$$

This completes the proof.

\section{References}

[1] E. Krätzel, Zweifache Exponentialsummen und dreidimensionale Gitterpunktprobleme, in: Elementary and Analytic Theory of Numbers, Banach Center Publ. 17, PWN-Polish Scientific Publishers, Warszawa, 1985, 337-369.

[2] H.-Q. Liu, On the number of abelian groups of a given order, Acta Arith. 59 (1991), $261-277$.

[3] —, The number of squarefull numbers in an interval, ibid. 64 (1993), 129-149.

[4] - On the number of abelian groups of a given order (supplement), ibid. 64 (1993), $285-296$

[5] S. H. Min, Methods in Number Theory, Vol. 2, Science Press, 1981 (in Chinese).

[6] P. G. Schmidt, Zur Anzahl Abelscher Gruppen gegebener Ordnung, J. Reine Angew. Math. 229 (1968), 34-42.

[7] P. Shiu, The distribution of cube-full numbers, Glasgow Math. J. 33 (1991), 287-295.

206-10, BAO GUO ST.

HARBIN, 150066

CHINA 